\title{
Transmission and prevalence of drug-resistant tuberculosis in a Brazilian setting under a directly observed therapy short-course strategy
}

\author{
Fábio Oliveira Latrilha ${ }^{[1]}$, Vera Simonsen ${ }^{[1]}$, Juliana Maira Watanabe Pinhata ${ }^{[1]}$, \\ Angela Pires Brandão ${ }^{[1],[2]}$, Vera Maria Neder Galesi ${ }^{[3]}$, \\ Eliseu Alves Waldman ${ }^{[4]}$ and Lucilaine Ferrazoli ${ }^{[1]}$ \\ [1]. Instituto Adolfo Lutz, Centro de Bacteriologia, Núcleo de Tuberculose e Micobacterioses, São Paulo, SP, Brasil. \\ [2]. Fundação Oswaldo Cruz, Instituto Oswaldo Cruz, Rio de Janeiro, RJ, Brasil. \\ [3]. Secretaria de Saúde do Estado de São Paulo, Centro de Vigilância Epidemiológica "Prof. Alexandre Vranjac", \\ Divisão de Controle da Tuberculose, São Paulo, SP, Brasil. [4]. Universidade de São Paulo, Faculdade de Saúde Pública, São Paulo, SP, Brasil.
}

\begin{abstract}
Introduction: We aimed to estimate the prevalence and transmission of drug-resistant tuberculosis in a high-burden Brazilian setting under directly observed therapy short-course strategy. Methods: Isolates of culture-confirmed pulmonary tuberculosis patients from Guarulhos, Brazil, diagnosed in October 2007-2011 were subjected to drug susceptibility and IS6110-restriction fragment length polymorphism testing. Results: The overall resistance prevalence was $11.5 \%$ and the multi-drug resistance rate was $4.2 \%$. Twenty-six ( $43.3 \%)$ of 60 drugresistant isolates were clustered. Epidemiological relationships were identified in $11(42.3 \%)$ patients; $30.8 \%$ of the cases were transmitted in households. Conclusions: Drug-resistant tuberculosis was relatively low and transmitted in households and the community.
\end{abstract}

Keywords: Brazil. Directly observed treatment. Drug resistance. RFLP. Transmission. Tuberculosis.

Brazil remains among the 20 countries worldwide with the highest number of tuberculosis (TB) cases ${ }^{1}$. The World Health Organization has recommended the use of the directly observed therapy short-course (DOTS) strategy to increase cure rates, reduce losses to follow-up, prevent the occurrence of new cases of drug resistance, and reduce the transmission of Mycobacterium tuberculosis complex (MTBC) strains ${ }^{2}$.

In 1996, the National Tuberculosis Program of Brazil (NTP) implemented the Emergency Action Plan for TB control. This plan initially prioritized 206 municipalities and was later expanded to 230 municipalities. Since then, the NTP has recommended the implementation of the DOTS strategy, mainly in the priority municipalities ${ }^{3}$.

\footnotetext{
Corresponding author: Lucilaine Ferrazoli.

e-mail: lucilaine.ferrazoli@ial.sp.gov.br

(D) https://orcid.org/0000-0002-5389-7056

Received 28 August 2019

Accepted 24 March 2020
}

Implementation of the DOTS strategy started in 1998 in Sao Paulo state. Between 2004 and 2011, the Surveillance Center of Sao Paulo state, in partnership with the United States Agency for International Development, intensified the DOTS strategy in Sao Paulo, Carapicuiba, and Guarulhos.

The emergence and spread of drug-resistant MTBC strains have threatened TB control globally ${ }^{1}$. Restriction fragment length polymorphism (RFLP) and spoligotyping have been applied in the study of TB transmission to improve public health surveillance $e^{4,5,6}$.

This study aimed to estimate the prevalence of resistance to first-line anti-TB drugs as well as the transmission of drug-resistant MTBC strains in a high-burden Brazilian setting using a DOTS strategy.

This cross-sectional study was performed in Guarulhos, a city with $>1.3$ million inhabitants ${ }^{7}$ and a TB incidence of $31 / 100,000$ inhabitants. Guarulhos has 67 primary health care units and one public health laboratory (PHLG) that performs smear microscopy and culturing. Mycobacteria cultures are systematically referred 
to the Adolfo Lutz Institute (IAL) for further identification, drug susceptibility testing (DST), and molecular typing. In Brazil culture and DST for TB are performed mainly for groups at high risk of TB resistance ${ }^{8}$.

For this study, the reference population consisted of all pulmonary TB (PTB) patients of both sexes aged $\geq 15$ years residing, diagnosed, and treated in Guarulhos between October 2007 and October 2011. Patients meeting these criteria, whose MTBC isolates were subjected to DST at the IAL, were enrolled in the study. Patients who received 24 supervised doses in the intensive phase and 48 doses in the second phase were classified as having completed the DOTS 8 .

The patients' sociodemographic and clinical information was collected from the TB notification system of Sao Paulo state (TBWEB). The laboratory results of smear microscopy and culturing were collected at the PHLG, while DST results were collected at the IAL.

MTBC isolates referred to the IAL by the PHLG underwent first-line DST (isoniazid [INH], streptomycin [SM], rifampin [RIF], ethambutol [EMB]) using a BACTEC Mycobacterial Growth Indicator Tube ${ }^{\text {TM }} 960$ system (Becton \& Dickson, Maryland, USA). Resistance to pyrazinamide was also detected using the pyrazinamidase test ${ }^{4}$.

Since 2007, the IAL has performed RFLP genotyping of all drug-resistant MTBC isolates received from approximately 80 laboratories in Sao Paulo state. RFLP was performed as previously described $^{4}$ and the patterns were analyzed by Bionumerics software (Applied Maths, Kortrijk, Belgium). Patients whose isolates presented identical numbers of DNA bands of the same molecular weight or one band difference were considered to be in a cluster, indicative of recent transmission. Isolates showing fewer than six IS6110 copies without an epidemiological link were excluded. RFLP patterns identified in this study were compared to all patterns of the Sao Paulo state database to include patients residing in Guarulhos but treated outside that city during the same study period.

The statistical analysis was performed using Epi-Info version 3.5 software (Centers for Disease Control and Prevention). Pearson's chi-square test with Yates correction or Fisher's exact test were used to examine categorical variables. Means were compared by the Kruskal Wallis test. $P$ values $\leq .05$ were considered statistically significant.

A total of 1,538 TB cases were identified in Guarulhos between October 2007 and October 2011. Of them, 1,280 (83.2\%) were PTB, 231 (15.0\%) were extrapulmonary TB (EPTB), 22 (1.5\%) were PTB associated with EPTB (PTB+EPTB), and $5(0.3 \%)$ were disseminated TB. Among the 1,302 PTB and PTB+EPTB patients (reference population), 476 (36.6\%) were culture-positive for MTBC. Of them, 407 (85.5\%) underwent DST (study population), which represent $31.3 \%$ of all PTB patients reported in the study period. Among these 407 PTB patients, 349 (85.7\%) were new cases and the remaining $58(14.3 \%)$ were previously treated for TB (Figure 1).

The reference and study populations showed similar characteristics regarding sex, years of education, and HIV coinfection. However, the study population was younger (mean $34.5 \pm 13.7$ years vs. $37.4 \pm 14.7$ years; $p<.001$ ), with higher frequencies of inmates $(26.5 \%$ vs. $9.8 \% ; p<.001)$ and retreatment cases $(14.2 \%$ vs. $7.8 \% ; p<.001)$.

Of the 407 patients, 335 (82.3\%) started DOTS, but only $54.1 \%$ (220/407) completed the course of at least 72 supervised doses.

The sociodemographic and clinical characteristics of the study population stratified by new and previously treated patients are shown in Table 1. Regarding the DST pattern, in the study population, 47 of $407(11.5 \%)$ patients showed resistance to at least one of the first-line anti-TB drug; of them, 17 (4.2\%) were multidrug-resistant TB (MDR-TB) and 15 (3.7\%) were INH-monoresistant cases (Table 1).

Among the new cases, the prevalence of resistance to at least one drug was $10.0 \%(35 / 349)$, while that of monoresistance to INH was 3.7\% (13/349) and that of MDR-TB was 2.6\% (9/349). Regarding previously treated patients, the prevalence of resistance was $20.7 \%(12 / 58)$ to at least one drug, 3.4\% (2/58) to INH alone, and $13.8 \%(8 / 58)$ to INH and RIF (MDR-TB). Compared to the new cases, previously treated patients were significantly more likely to be $\operatorname{MDR}(p<.001)$.

RFLP typing was performed on 43 of the 47 drug-resistant isolates because four did not grow in subculture. RFLP patterns were analyzed in two steps. First, the RFLP patterns of the 43 MTBC isolates from the study population were compared. Then, they were compared to all patterns in the RFLP database of drugresistant MTBC isolates of Sao Paulo state. In this second step, 20 additional patients residing in Guarulhos but treated in Sao Paulo city were included. Analysis of the RFLP patterns of these 63 patients showed that the number of IS6110 copies ranged from one to 19. Only seven isolates exhibited fewer than five copies. Two of them presenting one and three copies were classified as unique patterns. Of the remaining five isolates, each displaying four copies, two isolates had an epidemiological link and were kept in the study. The other three isolates were excluded from the analysis, resulting in $60 \mathrm{MTBC}$ isolates.

As shown in Figure 2, 26 (43.3\%) of the $60 \mathrm{~TB}$ cases belonged to five clusters (SP5, SP5W, SP1a, SP12b, and 4a). The SP5 cluster was the most prevalent, comprising $16(61.5 \%)$ patients, of whom $14(87.5 \%)$ had MDR-TB and two were INH-resistant. In one case, the SP5 pattern was mixed with another RFLP pattern (SP5 mix), probably due to laboratory contamination or mixed infection. Of the four remaining clusters, one comprised four patients and the other three comprised two patients each.

The sociodemographic and clinical characteristics of the clustered and unclustered patients were compared. Clustered patients presented similar frequencies regarding sex $(p=.738)$, age (range, 15-89 years, $p=.957)$, years of education $(p=.092)$, history of imprisonment ( $p=.677)$, HIV status $(p=.599)$, treatment history $(p=.468)$, treatment outcome $(p=.727)$, and sputum smear microscopy results $(p=.969)$. Compared to the unclustered patients, clustered patients were significantly more likely to be MDR $(69.2 \%$ vs $26.5 \% ; p=.002)$ and were under DOTS $(65.4 \%$ vs $29.4 \% ; p=.011)$. 


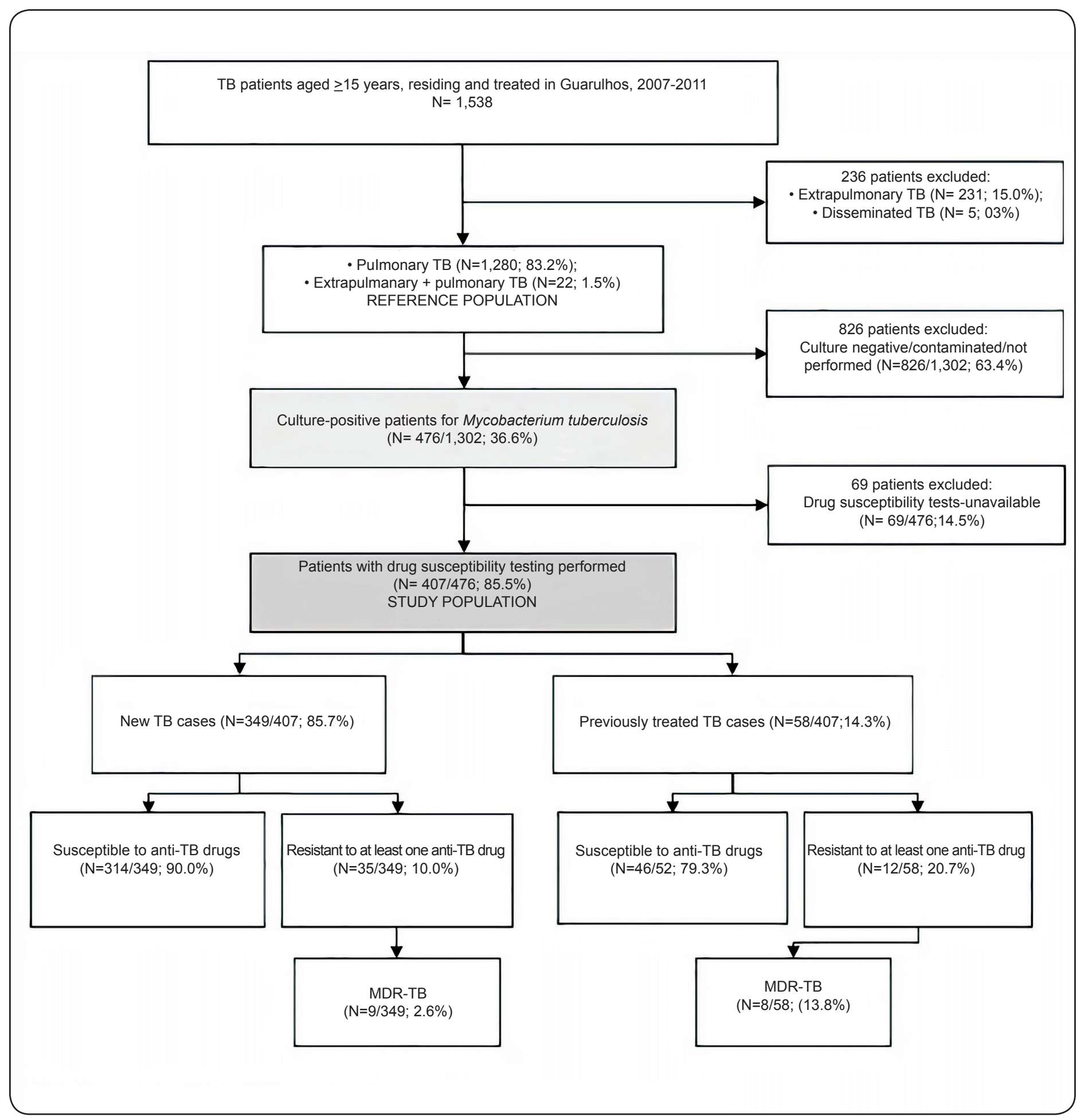

FIGURE 1: Study population selection, tuberculosis form, treatment, and drug susceptibility results of patients from Guarulhos, October 2007-2011. MDR: multidrug resistant; TB: tuberculosis. Anti-TB drugs tested included isoniazid, rifampicin, pyrazinamide, streptomycin, and ethambutol. 
TABLE 1: Characteristics of the 407 pulmonary tuberculosis patients according to treatment history, Guarulhos, October 2007-2011.

\begin{tabular}{|c|c|c|c|c|c|c|c|}
\hline \multirow[t]{2}{*}{ Characteristic } & \multicolumn{2}{|c|}{ Total (407) } & \multicolumn{2}{|c|}{ New cases ( 349 ) } & \multicolumn{2}{|c|}{ Previous TB (58) } & \multirow[t]{2}{*}{$P$} \\
\hline & $\mathbf{N}$ & $\%$ & $\mathbf{n}$ & $\%$ & $\mathbf{n}$ & $\%$ & \\
\hline \multicolumn{8}{|l|}{ Sex } \\
\hline Male & 302 & 74.2 & 256 & 73.4 & 46 & 79.3 & .424 \\
\hline Female & 105 & 25.8 & 93 & 26.6 & 12 & 20.7 & \\
\hline \multicolumn{8}{|l|}{ Age (yrs) } \\
\hline $15-19$ & 34 & 8.3 & 32 & 9.2 & 2 & 3.4 & .152 \\
\hline $20-39$ & 247 & 60.7 & 214 & 61.3 & 33 & 56.9 & \\
\hline$\geq 40$ & 126 & 31.0 & 103 & 29.5 & 23 & 39.7 & \\
\hline Mean age (yrs) & $34.6 \pm 13.713 .7$ & - & $34.2 \pm 14$ & - & $37.2 \pm 11.4$ & - & .003 \\
\hline \multicolumn{8}{|l|}{ Education (yrs) } \\
\hline $0-3$ & 46 & 11.3 & 42 & 12.0 & 4 & 6.9 & .371 \\
\hline $4-11$ & 257 & 63.1 & 226 & 64.8 & 31 & 53.4 & \\
\hline$\geq 12$ & 13 & 3.2 & 10 & 2.9 & 3 & 5.2 & \\
\hline Unknown & 91 & 22.4 & 71 & 20.3 & 20 & 34.5 & \\
\hline \multicolumn{8}{|c|}{ History of imprisonment } \\
\hline Yes & 108 & 26.5 & 96 & 27.5 & 12 & 20.7 & .353 \\
\hline No & 299 & 73.5 & 253 & 72.5 & 46 & 79.3 & \\
\hline \multicolumn{8}{|l|}{ HIV status } \\
\hline Positive & 27 & 6.7 & 21 & 6.0 & 6 & 10.3 & .348 \\
\hline Negative & 351 & 86.2 & 303 & 86.8 & 48 & 82.8 & \\
\hline Unknown & 29 & 7.1 & 25 & 7.2 & 4 & 6.9 & \\
\hline \multicolumn{8}{|l|}{ Treatment outcome } \\
\hline Cured/completed & 346 & 85.0 & 303 & 86.8 & 43 & 74.2 & .081 \\
\hline Lost to follow-up & 46 & 11.3 & 35 & 10.0 & 11 & 19.0 & \\
\hline Died of TB & 6 & 1.5 & 4 & 1.2 & 2 & 3.4 & \\
\hline Others & 9 & 2.2 & 7 & 2.0 & 2 & 3.4 & \\
\hline \multicolumn{8}{|l|}{ Treatment type } \\
\hline DOTS & 220 & 54.0 & 190 & 54.4 & 30 & 51.7 & .808 \\
\hline SAT & 187 & 46.0 & 159 & 45.6 & 28 & 48.3 & \\
\hline \multicolumn{8}{|l|}{ Sputum smear } \\
\hline Positive & 358 & 88.0 & 307 & 88.0 & 52 & 89.7 & .862 \\
\hline Negative & 35 & 8.6 & 29 & 8.3 & 6 & 10.3 & \\
\hline Not done & 14 & 3.4 & 13 & 3.7 & - & - & \\
\hline \multicolumn{8}{|l|}{ Drug resistance } \\
\hline Susceptible & 360 & 88.4 & 314 & 90.0 & 46 & 79.3 & $<.001$ \\
\hline Multidrug-resistant & 17 & 4.2 & 9 & 2.6 & 8 & 13.8 & \\
\hline Mono/polyresistant & 30 & 7.4 & 26 & 7.4 & 4 & 6.9 & \\
\hline $\mathrm{INH}$ & 15 & 3.7 & 13 & 3.7 & 2 & 3.4 & \\
\hline SM & 11 & 2.7 & 10 & 2.9 & 1 & 1.7 & \\
\hline $\mathrm{INH}+\mathrm{SM}$ & 4 & 1.0 & 3 & 0.8 & 1 & 1.7 & \\
\hline
\end{tabular}

DOTS: directly observed therapy short-course; HIV: human immunodeficiency virus; INH: isoniazid; SAT: self-administered treatment; SM: streptomycin; TB: tuberculosis. 


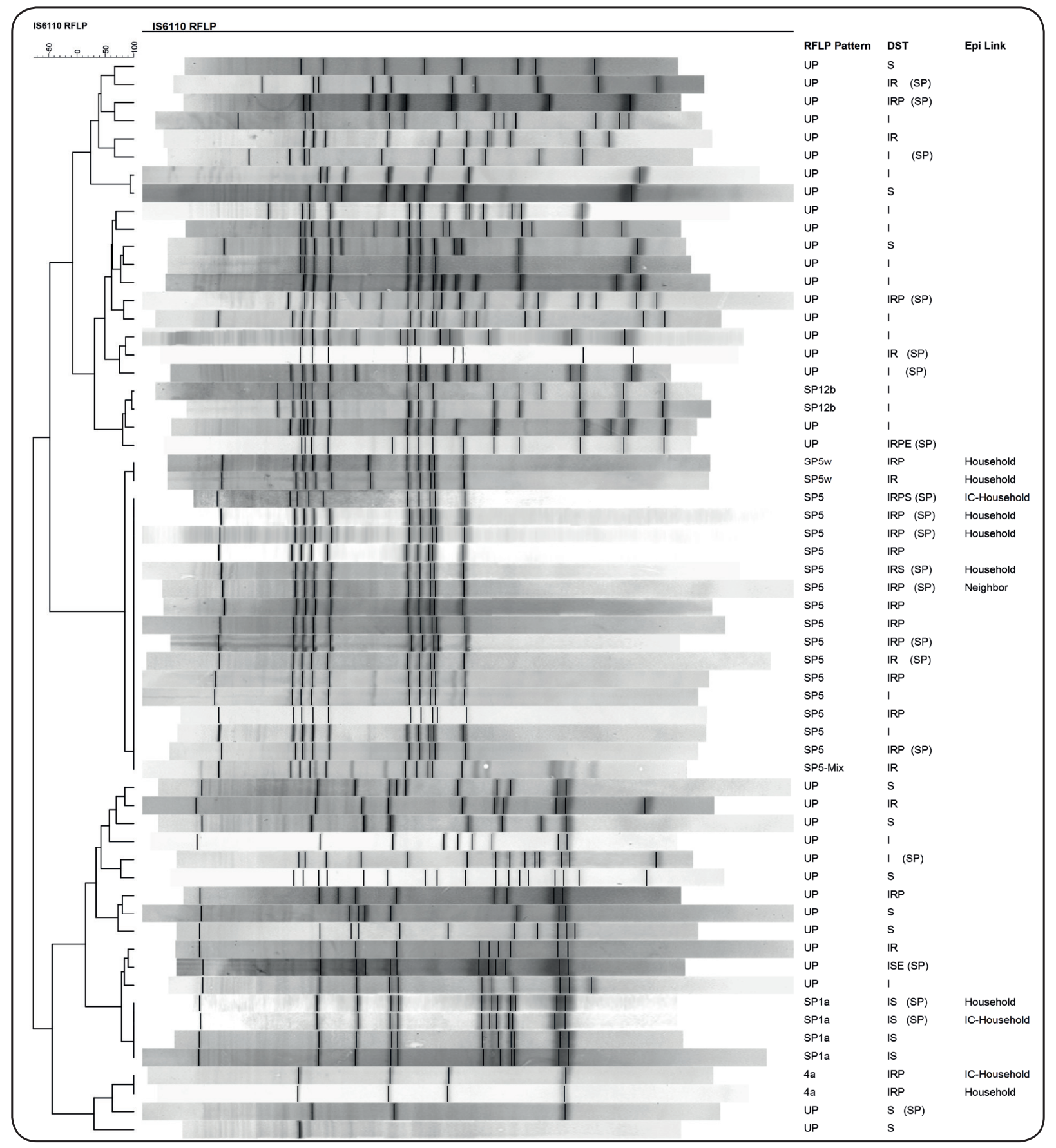

FIGURE 2: Restriction fragment length polymorphism patterns (RFLP) of 60 tuberculosis patients living in the municipality of Guarulhos, of whom 40 were treated in Guarulhos and 20 were treated in the municipality of Sao Paulo, October 2007-2011.

DST: drug susceptibility testing; E: ethambutol; Epi Link: epidemiological link; I: isoniazid; IC: index case; P: pyrazinamide; R: rifampicin; S: streptomycin; SP: patients treated in Sao Paulo City; UP: unique pattern.

Among the 26 patients belonging to clusters, epidemiological links were identified in $8(30.8 \%)$. All of these patients had household or neighbor contact with TB.

The drug-resistant TB prevalence found in this study is similar to those of previous studies performed in different states of Brazil. According to these studies, the overall prevalence of INH monoresistance and MDR for new cases was 3.3-4.9\% and $1.0-2.2 \%$, respectively ${ }^{4-9}$. The MDR-TB prevalence of $2.6 \%$ among new cases found in Guarulhos is higher than the 1.4\% found in Brazil during the II National Anti-Tuberculosis Drug Resistance Survey conducted in 2006-200710 and lower than the global prevalence (3.5\%) of MDR-TB/RIF-resistant TB reported in $2014^{11}$. In contrast with the drug resistance prevalence in new TB patients, the rates among retreatment cases had greater variability according to these studies. The rates of INH resistance and MDR were $4.3-17.3 \%$ and $12.0-16.7 \%$, respectively ${ }^{4-9}$. 
Almost half of INH-resistant or MDR-TB cases in our study occurred due to recent transmission. Previous studies highlighted the impact of DOTS on reducing transmission and incidence of drug-susceptible and -resistant TB in places with moderate and high rates of drug resistance. However, a study conducted in Taiwan demonstrated that, despite the reduction in drug-resistant TB rates, the prevalence of primary drug resistance remained stable during the 7-year study period ${ }^{12}$. In our study, trends in resistance or transmission prevalence were not assessed. However, 69.4\% of INH-resistant or MDR-TB patients had no previous history of anti-TB treatment, which may suggest that these patients acquired resistant TB due to recent transmission. In this study, the findings of primary drug resistance were corroborated by our molecular typing results. We found that $48.0 \%$ of the INH-resistant or MDR isolates belonged to cluster patterns, meaning that drug-resistant TB was recently transmitted among these patients.

The rate of monoresistance to SM was lower $(2.7 \%)$ than that to INH (3.7\%). All 11 SM monoresistant patients showed a unique RFLP pattern indicating the potential reactivation of a previous infection, since SM is not used to treat new cases. Similar findings were reported by Telles et al. ${ }^{4}$.

Epidemiological links were found for almost half of the clustered patients. Considering that there were three index cases, the transmission rate among cases with epidemiological links was $30.8 \%$. Except for one case (neighbor), all contacts were relatives, i.e., household transmission of drug-resistant isolates occurred frequently in this setting. The household transmission rate was similar to those found in studies performed in Brazil and elsewhere ${ }^{6-13}$ and could be explained by the fact that drug-resistant TB has a prolonged infectious period while the patient was under treatment.

The lack of epidemiological links among the majority of the drug-resistant cases (69.2\%) suggests ongoing transmission in the community. Studies conducted since the early 1990s using molecular typing have identified a significant proportion of transmission of drug-resistant strains in hospitals, prisons, or in the community ${ }^{4,5,6}$.

During the study period, Guarulhos improved the DOTS coverage: $82 \%$ of the patients started the DOTS course and 54\% completed 72 supervised doses compared with 1998-2004, when the DOTS coverage was lower $(40 \%)^{14}$. This proportion is higher than the national coverage of $38.3 \%$ for patients aged $\geq 18$ years reported by Reis-Santos and colleagues in $2011^{15}$.

There are several methodological limitations to our study. First, it was a retrospective study based on secondary data. Second, no formal evaluation was performed to ensure the accuracy of the DOTS information available on the TBWEB system. A comparative analysis between the study and reference populations showed no difference between them in the main clinicodemographic characteristics. However, there were higher numbers of inmates and retreatment patients in the study population, possibly because of intensification of culture and DST for people at risk of developing drug resistance. Finally, we did not genotype the susceptible MTBC isolates.
In conclusion, the prevalence of drug-resistant TB in Guarulhos was relatively low, and the disease was transmitted via household and community contacts. DOTS is an important strategy for reducing loss to follow-up rates and increasing cure rates, although additional efforts such as the rapid molecular diagnosis of drug resistance and improvements in contact investigation are needed to reduce the transmission of drug-resistant TB.

\section{ACKNOWLEDGMENTS}

We thank the tuberculosis surveillance team of the Municipality of Guarulhos as well as of the Municipal Public Health Laboratory of Guarulhos for authorizing access to the data.

\section{FINANCIAL SUPPORT}

This work was partially supported by the United States Agency for International Development (RFA 512-06-002).

\section{AUTHORS' CONTRIBUTION}

FOL: conceived and designed the study, performed the phenotypic tests, and collected laboratory data; VS: performed the genotypic tests; JMWP, APB, EW: analyzed the data; VMNG: acquired the patients' data; LF: conceived and designed the study, performed the genotypic and the data analyses.

\section{CONFLICT OF INTEREST}

The authors declare no competing interests.

\section{ETHICAL APPROVAL}

This study was approved by the Ethics Committee of the IAL (CCD-BM 19/2009) and the Department of Health Human Resources, Technical Division of Health Education Management of Guarulhos, Sao Paulo state.

\section{REFERENCES}

1. World Health Organization (WHO). Global tuberculosis report 2017. Geneva: WHO; 2017. 249 p.

2. World Health Organization (WHO). An expanded DOTS framework for effective tuberculosis control. Geneva: WHO; 2002. 20 p.

3. Hijjar MA; Gerhardt G; Teixeira GM; Procópio MJ. Retrospecto do controle da tuberculose no Brasil. Rev Saude Publica. 2007;41(Suppl. 1):50-7.

4. Telles MA, Ferrazoli L, Waldman EA, Giampaglia CM, Martins MC, Ueki SY, et al. A population-based study of drug resistance and transmission of tuberculosis in an urban community. Int J Tuberc Lung Dis. 2005;9(9):970-6.

5. Moss AR, Alland D, Telzak E, Hewlett D Jr, Sharp V, Chiliade P, et al. A city-wide outbreak of a multiple-drug-resistant strain of Mycobacterium tuberculosis in New York. Int J Tuberc Lung Dis. 1997;1(2):115-21.

6. Leung EC, Leung CC, Kam KM, Yew WW, Chang KC, Leung WM, et al. Transmission of multidrug-resistant and extensively drug-resistant tuberculosis in a metropolitan city. Eur Respir J. 2013;41(4):901-8.

7. Instituto Brasileiro de Geografia e Estatística. IBGE divulga as estimativas populacionais dos municípios em 01.07.2012 [Internet]. Rio de Janeiro: IBGE; 2012. Available from: http://www.ibge.gov.br/home/ estatistica/populacao/estimativa2012

8. Ministério da Saúde (MS). Secretaria de Vigilância em Saúde. Departamento de Vigilância Epidemiológica. Manual de recomendações para o controle da tuberculose no Brasil. Brasília: MS; 2011. 288 p. 
9. Micheletti VC, Moreira J da S, Ribeiro MO, Kritski AL, Braga JU. Drug-resistant tuberculosis in subjects included in the Second National Survey on Antituberculosis Drug Resistance in Porto Alegre, Brazil. J Bras Pneumol. 2014; 40(2):155-63.

10. Kritsky AL. Multidrug-resistant tuberculosis emergence: a renewed challenge. J Bras Pneumol. 2010;36(2):157-8.

11. World Health Organization (WHO). Global tuberculosis report 2014. Geneva: WHO; 2014. 154 p.

12. Chien JY1, Lai CC, Tan CK, Chien ST, Yu CJ, Hsueh PR. Decline in rates of acquired multidrug-resistant tuberculosis after implementation of the directly observed therapy, short course (DOTS) and DOTS-Plus programmes in Taiwan. J Antimicrob Chemother. 2013;68(8):1910-6.
13. Teixeira L, Perkins MD, Johnson JL, Keller R, Palaci M, do Valle Dettoni $\mathrm{V}$, et al. Infection and disease among household contacts of patients with multidrug-resistant tuberculosis. Int J Tuberc Lung Dis. 2001;5(4):321-8.

14. Villa TC, de Assis EG, Oliveira MF, Arcêncio RA, Gonzales R, Palha $\mathrm{PF}$, et al. Cobertura do tratamento diretamente supervisionado (DOTS) da TB no Estado de São Paulo (1998-2004). Rev Esc Enferm USP. 2008;42(1):98-104.

15. Reis-Santos B, Pellacani-Posses I, Macedo LR, Golub JE, Riley LW, Maciel EL. Directly observed therapy of tuberculosis in Brazil: associated determinants and impact on treatment outcome. Int J Tuberc Lung Dis. 2015;19(10):1188-93. 